\title{
Hacia la autonomía en Sanidad
}

\author{
Dra. MARIA CASADO \\ Directora del "Centre de Recerca Observatori de Bioètica i Dret" \\ Directora del Master de Bioética y Derecho \\ Profesora Titular de Teoría y Filosofía del Derecho \\ Universidad de Barcelona \\ Miembro de la Comision de Bioetica de Cataluña \\ Presidenta de la Asociación de Bioética y Derecho
}

INTRODUCCIÓN:

Las aportaciones de las ciencias biomédicas en el campo de la prevención, el diagnóstico y el tratamiento de las enfermedades, han cambiado la consideración no solo de lo que es la enfermedad sino, incluso, del concepto de muerte y entre la opinión pública coexisten tendencias de rechazo y de aceptación incondicional de los adelantos. Paralelamente, en los últimos años, las sociedades de nuestro entorno viven un importante proceso de revalorización de todo aquello que tiene que ver con la ética que, en el terreno de las ciencias de la vida, se manifiesta en la pujanza de la Bioética. Tanto es así, que a la tradicional exigencia de rigor científico le ha sucedido la reivindicación del análisis ético de las consecuencias de lo que se hace, $\mathrm{e}$ incluso de la misma actividad que se desarrolla. En este contexto se enmarca la aparición y el actual auge de la disciplina Bioética en las organizaciones sanitarias, ya que el cuidado de los aspectos bioéticos de la práctica hospitalaria incide de manera notoria en la mejora de la calidad, en el respeto de los miembros de los 
equipos por las cuestiones de confidencialidad y autonomía de los usuarios, y en la transparencia y justificación de las decisiones.

Las cuestiones bioéticas no son solo morales: la respuesta individual -moral- no es suficiente, se requiere de la reflexión colectiva puesto que de la respuesta que se adopte dependerá la forma de vida y de sociedad que generemos. Evidentemente el contenido de la reflexión bioética es materialmente "bio": las repercusiones en el terreno de la vida de los avances de las biotecnologías y de la biomedicina, que ocasionan conflictos para los que no siempre sirven las viejas respuestas dadas por los hombres a las grandes cuestiones sobre la vida y la muerte y las relaciones entre los hombres. No obstante, la Bioética tiene un alto contenido político que transciende a las meras implicaciones de la decisión ética o de la moral individual: las biotecnologías nos plantean dilemas cuya decisión implica optar por un estilo de vida frente a otros, por un modelo determinado de sociedad; lo que constituye evidentemente una opción política. Por otra parte, la Bioética requiere del bioderecho. Dado que los planteamientos y soluciones ante un problema pueden ser diversos, en ocasiones el acuerdo no se produce y es preciso que venga el Derecho a establecerlo. Entendiéndose aquí al Derecho como un sistema de organización social y de tratamiento de los conflictos. Este punto de vista ante el Derecho tiene en cuenta, mas que los aspectos meramente represivos, sus funciones promocionales y educativas, propias del estado de derecho actual.

Así concebida la Bioética se constituye en lugar de debate y reflexión sobre las biotecnologías desvinculado del discurso dogmático. Y parte de considerar como el único acuerdo general aceptable el que establece el derecho a discrepar, a no estar de acuerdo y a no imponer otro límite que el respeto a los Derechos Humanos, amparados por las constituciones y los instrumentos internacionales. Esta concepción de la Bioética, pluridisciplinar, plural y laica, insiste en la necesidad de racionalidad en la discusión. Para ello es necesaria la información que, en primer lugar, deben suministrar los científicos, que conocen los auténticos problemas 
puesto que trabajan materialmente con las tecnologías que cuestionamos. Después, con esos datos, la reflexión debe hacerse en común. Aportando cada una de las disciplinas sus específicas herramientas de análisis: antropológicas, sociológicas, jurídicas, éticas... Información y transparencia son requisitos necesarios para la democrática toma de las decisiones que a todos nos afectan. De esta manera, el análisis y la atención de los aspectos bioéticos en la actuación diaria genera hábitos de buena praxis y consenso sobre los valores que se han de promover y respetar.

\section{A PROPÓSITO DEL PLURALISMO Y LOS CAMBIOS SOCIALES}

Se ha señalado que nuestra sociedad es una sociedad plural. El pluralismo es un hecho en nuestra sociedad y, además, es un valor social y jurídicamente asumido como tal. No vivimos con un código de conducta y de valores homogéneo. No se existe un único modo de hacer y de valorar las cosas. No se aceptan criterios dogmáticos, pautas externas, ni morales vinculantes, válidas para todos; no contamos en la actualidad con un sistema de moralidad positiva, autoevidente, interiorizada por todos. Se admite que puede haber diversas maneras de ver las cosas y que - si no todas- algunas de ellas pueden ser legitimas y valiosas. La coexistencia de consideraciones diversas, incluso difícilmente compatibles, de un mismo asunto obliga a buscar el acuerdo a la hora de decidir, por eso se habla tanto de consenso y de establecer marcos procedimentales que permitan llegar a compromisos. Al mismo tiempo, del disenso, surge una demanda de legislación: puesto que no es posible el acuerdo, que lo establezca el derecho; se atribuye así al derecho un papel de zanjar la discusión. Es indudable que el ordenamiento jurídico tiene esta función de resolución de los conflictos, pero por otra parte es bien cierto que la existencia de una norma no dirime definitivamente la cuestión: el debate social permanece y la aplicación misma de la norma puede dar lugar a nuevos conflictos. 
El pluralismo fáctico, político y social, en que hoy nos hallamos inmersos, se opone a la idea de que exista un único proyecto válido, o de que pueda acaecer una armonía final que permita conjugar todos los distintos proyectos y finalidades de vida propugnados. Esta idea de que existe un solo modelo legítimo de convivencia, o de que en ultimo extremo todos son conciliables, -heredera de la tradición iusnaturalistasigue presente en el subconsciente colectivo y se hace especialmente evidente en la Bioética. Ello es palmario en los planteamientos confesionales, como es lógico, pero también subyace en gran parte de los que no se muestran como tales pero que comparten la idea de que La Verdad existe -aunque no necesariamente esta sea una verdad revelada-, y su conocimiento proporciona, además, criterios morales "naturales".

La historia nos ha mostrado, además, que después de elaborar un "modelo" suele darse un paso mas: el paso de tratar e imponerlo a los demás. Pero ese paso solo es legítimo si se logra mediante la persuasión, nunca por imposición. Por eso mismo, conviene señalar que nuestro planteamiento -que busca la convivencia de las opciones diversas, aceptando ante todo que lo son- tiene un paralelo útil para la comparación en el análisis de las transformaciones de la vida constitucional en los distintos países europeos: los cambios acaecidos en el concepto de Constitución, a causa de la existencia de grupos sociales con proyectos políticos y sociales diferentes y que han llevado a la propuesta de que la coexistencia se debe desarrollar en el ámbito de un compromiso constitucional "dúctil"y desde una consideración "flexible" del derecho.

Esta coexistencia de valores y principios en que se basa toda sociedad democrática debe ser asumida también en el terreno de la argumentación etico-jurídica aplicada a las biotecnologías y a sus aplicaciones biomédicas. Para ello, cada uno de los valores y principios se concibe no con un carácter absoluto, sino de manera compatible con los demás valores y principios con los que ha de convivir. Este sería un "modelo europeo", enemigo de la imposición por la fuerza, y se configuraría como una propuesta política que exige actitudes moderadas, a la 
vez que constructivas y positivas, y una actitud mental y vital posibilista. El único contenido 'sólido' de la ciencia de una Bioética pluralista debería defender rigurosa y decididamente es el de la pluralidad de valores y principios, y no el predeterminar rígidamente el modo en que esos valores y principios han de convivir. Así se trataría de salvaguardar los distintos valores y principios entendiéndolos no como absolutos sino permitiendo que se respeten todos en la medida de lo posible. Como es uso en la jurisprudencia constitucional que, mediante una ponderación de principios, valores y derechos, evita la prevalencia apriorística de cualquiera de ellos, jeraquizándolos sólo en cada caso concreto.

Naturalmente la flexibilidad conlleva un cierto grado de imprevisibilidad, que no es grata para los que desean la certeza absoluta en las decisiones, los que buscan "recetas morales" siguiendo las cuales se sienten cómodamente seguros. Ahora que estamos ya entrando en el S XXI, parece llegado el momento de dejar los andadores y asumir las decisiones que nos corresponde tomar a nosotros mismos.

\section{LA AUTONOMÍA DE LOS PACIENTES Y EL CONCEPTO DE SALUd}

Actualmente el reconocimiento de la autonomía de las personas cuestión central en el ámbito moral y en el jurídico- va extendiéndose en nuevos campos. Así sucede en el terreno de la sanidad, tradicionalmente regida por el principio de beneficencia y por la idea de que el médico -como experto en enfermedades- era el que tomaba las decisiones, ya que era quien sabía del asunto. Las relaciones sanitarias experimentan importantes cambios derivados de múltiples factores; uno de ellos es la masificación e impersonalización en que desarrollan su actividad los grandes hospitales, que han contribuido al deterioro de un modelo de relaciones medico-paciente basadas en la confianza y obligan a no presuponer cual es la opinión de ninguna de las partes implicadas en la misma -dado que la relación se desarrolla entre desconocidos cuya je- 
rarquía de valores no puede ser presupuesta ya que, como se ha dicho, la sociedad de que ambos provienen no es homogénea-. A esto se añade que el concepto mismo de salud ha ido cambiando de estar centrado en la ausencia de enfermedad ha pasado a pivotar sobre la idea del bienestar de los sujetos, como hace la definición de la OMS. Ello implica que sea el paciente mas indicado para definir su propio bienestar que un "técnico externo" (como sucedería si el eje estribase en la enfermedad)

Naturalmente el movimiento a favor de la autonomía de las personas es algo que trasciende el mundo de la sanidad: se constituye en el rumbo general civilizatorio y es la señal de madurez de personas y sociedades. De la heteronomía a la autonomía. Necesariamente es así en el terreno de la ética, pero también en el del derecho y la política: de la asignación de estatus a la autonomía de la voluntad, de la dictadura a la democracia. El derecho al voto, la libertad de residencia, de pensamiento y de expresión, de elección de pareja, ... la consolidación del reconocimiento de los derechos de las personas se encamina a establecer parcelas de libertad cada vez mayores a los sujetos y no es raro que también sea así en el mundo sanitario. Posteriormente, las asociaciones de consumidores y enfermos han desempeñado un papel fundamental a la hora de la aceptación de las cartas de derechos de los pacientes, pero cabe señalar que la citada definición de salud no ha resultado ajena al cambio de mentalidad a que estamos asistiendo.

Por otra parte, si bien es cierto que el movimiento a favor de la autonomía de los pacientes junto con la reivindicación de una sanidad equitativa para todos los ciudadanos, constituyen el marco en que se han ido desarrollando los grandes cambios de la sanidad, no lo es menos que ha sido la aplicación de la biotecnología a la sanidad y el consiguiente auge de las ciencias biomédicas los que han dado lugar a una transformación tan importante en el mundo sanitario que ha puesto en cuestión los objetivos tradicionales de la medicina, la demanda sanitaria, la investigación, e incluso la priorización de los gastos en los presupuestos estatales. 
Además, en el terreno de las aplicaciones biomédicas existe otro puntal que junto a los objetivos de la medicina puede proporcionarnos orientaciones fiables: la consideración de los cambios habidos en el mismo concepto de salud. La definición de salud habitualmente utilizada se debe a la Organización Mundial de la Salud y su generalización ha dado lugar a cambios importantes en el entorno sanitario al pasar de centrarse en la ausencia de enfermedad a referirse al bienestar del sujeto. $\mathrm{Si}$, siguiendo a la OMS, aceptamos considerar la salud como "el estado de completo bienestar físico, psíquico y social", la consecuencia inmediata es que es precisamente el sujeto interesado quien puede determinar su estado de salud. El alcance de este cambio de planteamiento aún no se ha acabado de manifestar, pero la primera de todas las consecuencias extraibles -la más evidente y la más 'revolucionaria' en el mundo sanitario-, ha sido desplazar el centro de decisión del medico al paciente: de la beneficencia a la autonomía. Si bien hay que advertir que hacer pivotar en la autonomía las relaciones sanitarias es algo que dista de haberse consolidado, el análisis y la atención de los aspectos bioéticos en la actuación diaria genera hábitos de buena praxis y consenso sobre los valores que se han de promover y respetar.

\section{LOS ÚLTIMOS AVANCES NORMATIVOS}

La libertad, la igualdad, la justicia y el pluralismo como valores constitucionales en los que se asienta la convivencia social, son también principios rectores de la Bioética y los derechos de los pacientes pueden ser considerados como parte integrante del despliegue de derechos derivados de los Derechos Humanos. La Ley General de Sanidad, en su artículo 10, promulga un conjunto de derechos que conciernen a las administraciones públicas en lo que hace a las prestaciones que deben establecerse, pero que también resultan vinculantes en el terreno de los servicios sanitarios privados en todo lo demás. Además, a partir del ar- 
tículo 43 de la Constitución que establece el llamado derecho a la salud, se confiere a los poderes públicos la organización y tutela de la salud pública mediante la prestación de los correspondientes servicios y el establecimiento de medidas preventivas.

Las cartas de derechos de los pacientes y usuarios se han consolidado como herramienta para garantizar en la mayor medida posible el cumplimiento de los derechos reconocidos en la legislación. Para ello toman en consideración: el respeto a la autonomía y libertad del paciente, a la intimidad y la confidencialidad -en sus distintas facetas como por ejemplo en referencia a la historia clínica- la protección ante la investigación o ante cualquier posible discriminación y, naturalmente, al correcto funcionamiento de los servicios asistenciales, con el grado de calidad requerido. De la mayor importancia son los derechos que se concretan en el llamado consentimiento informado, entendiendo por tal el derecho a ser informado y a elegir entre las distintas opciones disponibles con el necesario conocimiento de causa, con las consabidas excepciones de riesgo para la salud publica, falta de capacidad o urgencia que tanto juego han prestado a la literatura especializada en torno al tema. Es importante, además, tomar conciencia de que la tendencia general se encamina hacia la mayor aceptación de los efectos de la autonomía, tanto en lo que hace a la admisión de decisiones previas y testamentos vitales como en lo que hace a la toma en consideración de la voluntad de los menores en la medida de lo posible, según su grado de madurez.

Las nuevas cartas de derechos de los pacientes y usuarios de los servicios sanitarios constituyen un ejemplo de la labor que se esta llevando a acabo y que incide de manera directa en el proceso de consolidación del efectivo ejercicio de la autonomía de los pacientes a que nos estamos refiriendo. En este sentido, el reconocimiento de los derechos de los usuarios del sistema nacional de salud vincula a las administraciones públicas pero, en la mayoría de los casos, pueden ser ejercidos también en el ámbito privado. Incluso pueden ser exigidos por la Administración como requisito para la acreditación de los centros, como de 
hecho ya lo hacen importantes entidades de privadas cuyos estándares de calidad incluyen numerosos protocolos indicadores del respeto a la autonomía y los demás derechos de los pacientes. Generalmente, las cartas de derechos se refieren al entorno hospitalario pero, cada vez mas, se toma en cuenta su aplicación en el terreno de la asistencia primaria.

Ejemplos como la nueva carta de derechos y deberes de los pacientes elaborada por Servei Catalá de la Salut, o la existente en los Servicios Vascos de Salud, establecen los derechos básicos ligados a los derechos fundamentales y a la dignidad humana. Así sucede con los derechos que se derivan de la autonomía del paciente -información, consentimiento, confidencialidad-, de la igualdad y no-discriminación, o de las prestaciones legalmente establecidas. Además, estos se completan con el reconocimiento de los derechos específicos como usuario del sistema asegurador -elección de profesional, tiempo de espera adecuado a la patología, calidad de los servicios...-. También se incluyen derechos referentes a los avances biotecnológicos, como ya hace el Convenio de Derechos Humanos y Biomedicina: trasplantes de órganos, investigación, tecnologías genéticas, aparecen ya contemplados en las mas recientes declaraciones.

Así se observa también en dos textos normativos que están de plena actualidad: la reciente Ley sobre los derechos de información y autonomía del paciente y documentación clínica establece una regulacion estatal armonizada en este terreno, sobre la base de lo regulado ya en diversas autonomías, y, la Carta de Derechos Fundamentales de la Unión Europea que -aunque carente, por el momento, de valor jurídico en sentido estricto- parece llamada a desempeñar un importante papel, si reparamos en la voluntad política del Parlamento y del Tribunal, cuanto menos.

La nueva norma catalana se basa en la importancia considerar los derechos de los pacientes como eje de las relaciones clinicoasistenciales y tiene por objetivo profundizar en la concreción práctica de los derechos a la información, consentimiento y acceso a la documentación clínica. 
Para ello contempla el alcance del derecho a la información y determina que la titularidad del mismo corresponde al paciente, quien tiene derecho a poseer toda la información pero también a que sea respetado su deseo de no ser informado, si es esa su voluntad. Se requiere el consentimiento libre e informado para cualquier intervención en el ámbito de la salud, regulándose la documentación del mismo y sus excepciones. Por primera vez - tras su inclusión en el Convenio de Oviedo- se introduce en nuestra normativa no solo la posibilidad de otorgar el consentimiento por substitución, sino la de establecer voluntades anticipadas; se enumeran los requisitos del documento de directrices previas y se establece que sea incorporado a la historia clinica del paciente. También se reconoce el derecho a la intimidad y la obligación de confidencialidad en el tratamiento de los datos y, tras definir la historia clínica como conjunto de documentos relativos al proceso asistencial del enfermo, establece que deberá poseer el máximo grado de integración y que deberá garantizarse su seguridad y correcta conservación; así mismo, se delimita el contenido de la historia y sus usos; además, instaura el derecho del paciente de acceder a la historia clinica y propone la creacion de un registro unico en el cual consten también las voluntades anticipadas si existen. Como puede deducirse se trata de un texto pionero que supone un paso importante en cuanto a la aceptación de las tesis que aquí se sostienen.

En este mismo sentido, si bien con un nivel distinto de vinculatoriedad y alcance territorial, conviene mencionar que la Carta de Derechos Fundamentales de la Unión Europea contempla nada menos que en sus artículos 1, 2, 3 y 8 la inviolabilidad de la dignidad humana, el derecho a la vida y a la integridad, concretando que en el marco de la medicina y la biología se respetarán en particular el consentimiento libre e informado de la persona, la prohibición de prácticas eugenésicas, la utilización del cuerpo humano como objeto de lucro, la clonación reproductora, y la protección de los datos de carácter personal. Por otra parte, el articulo 35 establece la protección a la salud indicando que toda perso- 
na tiene derecho a la prevención sanitaria y a beneficiarse de la atención sanitaria en las condiciones establecidas por las legislaciones nacionales; precisando que, al definirse las políticas y acciones de la Unión, se garantizará un alto nivel protección a la salud humana.

\section{CONCLUSION}

Puede afirmarse, pues, que se están produciendo importantes transformaciones en el mundo de la sanidad y la investigación de las que aún no se ha tomado plena conciencia. Pueden ilustrar las anteriores afirmaciones cambios de envergadura como los ocasionados por la exigencia de respeto a la autonomía y la libertad individual: desde la introducción de las políticas de promoción del consentimiento informado, a la paulatina asunción de los testamentos vitales y directrices previas, como se ha puesto de manifiesto. También existen ejemplos muy señeros que atañen al principio de igualdad, como la priorización de los gastos sanitarios, las modificaciones de los listados de prestaciones de medicamentos con cargo a la seguridad social o el acceso a la medicina genética.

Por todo ello, se puede afirmar que el respeto y la promoción de los Derechos Fundamentales, reconocidos constitucionalmente, son un criterio válido a la hora de establecer políticas sanitarias y áreas de investigación prioritarias. En Bioética el acogerse a estos criterios representa una guía fiable ya que proporcionan la mejor pauta a la hora de juzgar la bondad y maldad de las biotecnologías y las prácticas biomédicas. Ellos representan el consenso logrado en torno a cuáles son las exigencias de la dignidad humana.

Así, parece lícito afirmar que con el cambio de milenio se está consolidando un cambio cultural importante: pivota sobre el eje de la libertad y la responsabilidad y se encamina a intentar asegurar el mayor respeto a la dignidad de las personas. 
En este texto se resumen las ideas básicas expuestas en el capitulo 1 del libro "Wistudios de bioetica y derecho" de la editorial Tirant lo Blanch, Valencia, y se reelaboran algunos argumentos recogidos en el discurso de pronunciado en la Real Academia de Medicina de Murcia, con motivo de lu recepción como académica correspondiente. 